International Journal of Engineering \& Technology, $10(1)(2021) 72-80$
SPC
International Journal of Engineering \& Technology
Website: www.sciencepubco.com/index.php/IJET
Research paper

\title{
Health tracker: data acquisition and analysis for monitoring health trends and assessing disease risk
}

\author{
Du'a Alzaleq $^{1 *}$, Suboh Alkhushayni ${ }^{2}$, Austin FitzGerald ${ }^{3}$ \\ ${ }^{1}$ Minnesota State University Mankato \\ ${ }^{2}$ Minnesota State University Mankato \\ ${ }^{3}$ University of Wisconsin Platteville \\ *Corresponding author E-mail: dua.al-zaleq@mnsu.edu
}

\begin{abstract}
This paper describes HealthTracker, a mobile health application to record, store, display, and analyze personal health data. This application allows an individual to log several types of data encompassing their personal health. HealthTracker serves as a model for both a recording and a recommending system. Its goal is to serve as a bridge for future personal health systems to build from. A person's health information is displayed in an easy-to-understand manner but is also practical for medical professionals. Users should find the system useful and effective no matter if they use it simply or extensively. Currently, the system serves as a prototype for determining the practical applications for smart health systems running on mobile platforms.
\end{abstract}

Keywords: HealthTracking, Chronic Diseases, Caregivers, Health Informatics, risk assessment

\section{Introduction}

Victims of disease must regularly record physiological data in order to monitor their healthstatus. However, any individual may have an interest in monitoring their health status or long- term risk for chronic diseases. Simple physiological data measurements can show health trends over time, and the same data can beused to calculate risks for many major diseases. The software applications used in clinical settings to assess risk are not typically available for the general public, nor for patient home use. For thatreason, we have developed HealthTracker, a mobile application that allows for physiological data entry, display, and analysis in a package forboth medical patients and healthconscious people. HealthTracker aims at providing users multiple ways of recording and monitoring various aspects of their overall health. For example, daily user weight can be recorded quickly and frequently. All health records can beviewed for specific dates or shown statistically as

averages for a chosen period. The system also generates charts and tables to compare data types.Microsoft HealthVault is used as a cloudbased storage solution, where a user or caretaker can monitor data remotely. With these quantitative health records stored through HealthTracker, thesystem can estimate their risk of developing multiple chronic diseases such as cardiovascular disease (CVD) and subtypes of it as well as diabetes and fatty liver disease (FLD). This can all be done without making appointments forclinic-based diagnostic testing, saving the user time, money, and effort. Self-monitoring of personal health trends has been shown to improvehealth in people with and without chronicillnesses, as well as increase long-term health engagement (Kim, et al., 2016).

HealthTracker also functions as a diary for food intake, physical activity, medication logging, as well as any miscellaneous entries a user wishes tomake. In a study on the use of a diary system to promote self-monitoring and health-related self- efficacy, it was stated that "The 8-week daily diary intervention was associated with improved exercise and healthy eating identity, and simultaneously improved exercise and healthyeating self-efficacy in the intervention group when compared with the control group." (Urzi, etal., 2016). These diary entries can provide usefulcomplementary information to the quantitative data types mentioned in the preceding paragraph;one who wishes to monitor their weight would also likely find it favorable to record their caloricintake. A caretaker who wishes to track a patient's medication intake could do so usingHealthTracker.

Following this section of the paper, we show howour application compares to similar existing ones.Afterward, we list the requirements specificationsenvisioned during the initial stages of oursystem's development. The design of the system is then explained; this includes the system cohesion, the user interface and how every section of the module is related to each other. Delving further into detail on the mechanics of the system, we describe how we accomplished the functionalities of the system, theimplementation. Concluding the paper, we discuss our plans to further advance the vision ofHealthTracker. 


\section{Related work}

Other systems currently exist that perform individual tasks that HealthTracker is capable of,but none integrate all major parts of which HealthTracker does: quantative health data, diet, physical activity, and medication logging as wellas chronic disease risk calculation. One such system, titled iCare, uses wireless sensors to monitor health factors of the elderly. It consists ofa personal health monitoring system that can be utilized by caretakers. While HealthTracker is also capable of detecting critical conditions for elderly persons, it is not restricted to this specificage group and contains a broad and tailorable setof data types that are not restricted by physical body sensors. All data types may be toggled on oroff in the interface. That is, by utilizing a combination of pre-determined data types as wellas any additional health information chosen by the user, the system can accommodate for many health monitoring scenarios. Another system named BeWell was developed to promote healthier lifestyles of its users. In short, it tracks physical activity, sleep patterns, and socialinteractions and provides feedback andrecommendations pertaining to these three aspects of a person's health. There are few applications composed of data recording,logging, and disease risk calculators availablecurrently. The goal of HealthTracker is not to only store and display data, it is to take part in analyzation of the data - to remove some burdenfrom the user.

\section{Requirements specification}

HealthTracker is a mobile system that operates inunison with Microsoft HealthVault. HealthVault is a system that allows for health information tracking but requires users to have an establishedinternet connection and access to a desktop computer; mobile HealthVault applications onAndroid and iOS devices were discontinued as ofJanuary 2018. To make health data recordingeasy and available, HealthTracker allows users torecord any health information at any time from their mobile device, which is automatically synced with their Microsoft account. Furthermore, some patients whom require strict health monitoring are not in a capable state of doing this by themselves, which defines the needof caregivers. Likewise, the system supports multiple user accounts on the same device, meaning caregivers can record the health credentials of several individuals, making it easier to care for multiple medical patients. Of course, different people can also log into HealthTracker with their individual Microsoft account, all on the same smartphone. This fills therequirement pertaining to a family or shared device situation where multiple people wish to monitor their health but don't all have access to adevice. Every time a user accesses a different account recorded on the device, they must provide the username and password associated with the Microsoft account, maintaining securitypersonal health information security. Once a usersuccessfully logs into the system, they have an arsenal of health tracking/health analysis functionalities at their disposal, which are described below.

\subsection{Caregiver data analysis}

Unless properly trained, individuals are not likelyable to identify chronic disease risk factors with their own health data. HealthTracker's data analysis system provides caregiving functionalities, making it possible for anyone equipped with the application to be a caregiver. Health information may be entered for the systemoperator, themselves, in which case this person ismonitoring their own health statistics. Similarly,someone that requires health monitoring but isn'tin a suitable position to interface with the application may be cared for with the help of HealthTracker and a designated caregiver.

Any person burdened with a chronic disease mayrequire this type of assistance. Moreover, the person in need may simply be in an aging, fragilestate where their health statistics drastically affect their physical condition in a short amount of time.Either way, the vitals, or health information, of this person, can be frequently analyzed by HealthTracker, which will output results to a caregiver, for any changes or trends in order to detect the presence of certain fatal conditions. Inthe case of a more minor risk scenario, HealthTracker allows users to act as caregivers on their own. They can continuously enter their updated health information and use it to assess their risks for certain conditions. They may also simply view data trends and, consequently, alter or continue certain lifestyle habits that directly affect their health information they monitor.

In the past, for someone to be an effective caregiver, they needed knowledge of the disease or condition they were caring for. Presently, HealthTracker allows someone to function as an effective caregiver without being as knowledgeable of their patient's diagnosis. The role of this type of caregiver is to use the application's functions to closely monitor the behavioral trends of health information of the person they're caring for. If while viewing agraph of health information or calculating a risk percentage, for example, the caregiver notices an indicative characteristic of a dangerous situation,provided by the system, the caregiver can then urgently take the next step in treating or caring forthat person's condition. To be an effective assistant in caring for a person in a critical healthstate, the caregiver only needs the ability to run the system and an awareness of how to respond to a critical state of the patient.

HealthTracker can also sufficiently operate inprofessional medical settings, if needed. Atrained caregiver, nurse, or physician will have more access to someone's health specifications than a user of the system in a private setting. Thisis obviously assumed and does not encompass exceptional circumstances in which, for example, a user of HealthTracker in a private setting utilizes a smart device that accurately retrieves their blood pressure, blood glucose, or heart rate.Although, most medical professionals are able toeasily access a patient's health information, meaning they can use HealthTracker as a risk calculating device for whichever chronic diseasethey are searching for.

\subsection{Personal health information system}

Our system offers users the option to enter whatever personal health information they please.Basic information consists of fields such as age, gender, weight, and if the individual is a smoker.More advanced fields include systolic and diastolic blood pressure, HDL and LDL cholesterol, and blood glucose levels. The user can view their health information within the mobile application, making this functionality widely available, or by accessing their MicrosoftHealthVault account on any device of their choosing. One of the main functionalities of Microsoft HealthVault is to provide users a safe,easy way to store their medical information and personal data, compared to other online vaults that have experienced data breaches in the past. However, Microsoft discontinued their mobileapplications that allowed for this exact feature, HealthVault for iOS and Android. While planning and designing other features for HealthTracker, we also intended to continue allowing mobile users with a MicrosoftHealthVault account the ability to record andaccess their health information from their smart device. HealthTracker makes both of thesepossible with an easy to maneuver/easy to understand user interface. A user can choose to update their health information, followed by further guidance and descriptions from thesystem pertaining to what information they are currently entering/updating. A user may also simply choose to access and view their health information for other purposes besides updating. 


\subsection{Assistant}

HealthTracker also offers the functionality of a user assistant. Along with recording information related to the user's physical body, the system allows for the logging of fields such as foodintake, exercise, and personal medications. By letting users record and review their daily habits,the system functions as a motivating assistant, pushing the user to develop a healthier lifestyle. Users may frequently check, for example, their calorie intake, receiving feedbackon their eating habits for a particular day. A usermay notice an unhealthy amount of food or specific nutrient intake for a certain day or set ofdays, making them aware of changes they shouldmake to their diet. The system also functions as an active assistant, if the user so chooses, rather than just providing the user with additional information besides their previously mentioned data related to the body. Specifically, the application features a reminder system that the user can program to display notifications to theirmobile device at certain intervals or for certain times of the day. A common example of a systemreminder would be to prompt the user, from the main notifications list of the mobile device, to take their daily medications, which the user high risk for diabetes, for example, they may be propelled to consult further medical assistance toensure the state of their well-being.

\subsection{Accessibility}

The system encompasses global accessibility standards to allow use by as many people as possible. Measurement units for all data types can : would have already entered the specifications for in the system.

\subsection{Risk assessment/guidance}

The primary goal of our research included locating methods for determining someone's riskfor a chronic disease. We directed our focus on six different conditions: hard coronary heart disease (HCHD), cardiovascular disease (CVD), diabetes, fatty liver disease, hypertension, and atherosclerotic cardiovascular disease (ASCVD).After we acquired information on these diseases, we discovered that CVD encompasses hard coronary heart disease, ASCVD, and hypertension. CVD is a general state of someone's circulatory system that indicates an increased risk for any, or all, of these three conditions. HCHD, CVD, and ASCVD are all technically diseases in and of themselves but can be grouped under a general term known as cardiovascular disease. It can be stated that HealthTracker calculates for three different conditions, which may include a combination of up to five different diseases. A user has the optionto calculate their percent risk for developing a disease within a specific timeframe. Additionally,in the system, there exist multiple ways for calculating a certain disease, which determines the length of the timeframe for developing the disease. The calculation method, chosen by the user, relates to different organizations that conducted and documented studies on chronic diseases. These research groups are how we obtained the necessary information and processes to build the risk calculation system ofHealthTracker. We combined the findings of these research facilities into a single unit to assessan individual's percentage for developing the disease. This functionality of HealthTracker serves as an assessment tool, as well as a guidance

be chosen individually. Glucose is measurable in both $\frac{m m o l}{L}$ or $\frac{m g}{d L}, \mathrm{HbAlc}$ in DCCT (\%) or IFFC $\left(\frac{m m o l}{m o l}\right)$, cholesterol in $\frac{m m o l}{L}$ or $\frac{m g}{d L}$, weight in kilograms or pounds, and length in centimeters or

tool. If a user discovers they are at a inches. Similarly, a user can choose their preferred date format: day/month/year or month/day/year. In order to be accessible for people of varying backgrounds, the system also works for Android devices running version 4.4.xand above; over $88 \%$ of all Android devices are able to run this application (citation). The app also has support for multiple users, so that healthrecords can be kept for multiple people using thesame device.

\section{System design}

We will now describe the design of our system. We can separate the system into three main categories: health information, assistant/data logging, and risk assessment. Each of these is accessible from the main menu of the mobile application, run on a smartphone. We will furtherseparate the system design section into four categories: first describing the architecture and usability of running on a smartphone, followed bydata, HealthVault, and risk assessment designtechniques, in consecutive order.

\subsection{Smart device}

HealthTracker runs on any android device with aminimum API requirement of 19, which was released with Android 4.4 KitKat. This version ofAndroid was released in 2013, meaning our system should be able to run on most smartphonedevices that are being used today. In fact, at the time of this research, over ninety-six percent of all Android devices currently in use meet this minimum API level requirement (Singh, 2018).

a) Architecture

The main menu of the system consists of three groups of buttons. The first group, contained in the center of the screen, allows a user to enter or update their health information. The types of information that a user can access, which is doneby selecting one of five labeled buttons, are weight, glucose, blood pressure, cholesterol, andthyroid. The second group of buttons, located in-line at the bottom of the screen, allow users to view the trends and histories of their data entries.They may access their logs, a chronological record of all health information entered by the user; charts, graphs of health information; diary, additional events, reminders, or information the user has recorded in their calendar; statistics, theaverage values for health information entries overa designated time period; or risks, their percent chance of developing chronic diseases. There is also a settings button, located on the very right ofthis second group of buttons, which allows a userto manage currently linked Microsoft HealthVault accounts or to choose the measuringsystem for their health information (pounds or kilograms for weight, for example).

Each separate menu for health information entriesconsists of labeled text fields, along with a "save"and "cancel" button. When a user chooses to viewa certain type of data trend, they are prompted tochoose what health information they would like to view trends, graphs, or risks 
for. The system then, depending on what functionality the user has chosen, displays and labels the desired healthinformation in whatever format the user chose.

b) Usability

Our goal of using a simple yet descriptive buttonlayout was to make the system relevant to as many people as possible. In other words, anybodycan navigate through the various menus of the application, and they may also do just this if theyplease. However, for anyone that is looking to getthe most, or even some, out of Health Tracker's functionalities, we made sure to clearly label anddocument each menu of the system. This allows the user to know exactly where they currently arein the system, what their capabilities are pertaining to that certain interface, and how to utilize the capabilities of the interface. The twomain options stemming from the main interface of HealthTracker are entering health information and viewing this health information or analyzations of this information.

\subsection{Data}

Data presentation in HealthVault is done in five separate ways. The method of display depends onwhat type of data a user wishes to view and whatmethod the user wants to use for data presentation. All five types of data display are described below.

a) Diary

The diary option is used for displaying health information, which refers to personal, numerical data related to the user's body that they have entered in the system. From the top of the screen,the user may select which category of healthinformation to view. Within each category, the diary displays a list of all data entries performed by the user, in chronological order. For eachentry, data values are included, along with the date of the entry.

b) Charts

The charts option also displays health information, except this option does so in a graphical manner. Again, a user chooses which health information category to view a complete graph of. Not only do they have the choice of onespecific type of information, but they may also choose from pre-selected combinations of healthinformation, which will display the graph of bothtypes of health information.

c) Statistics

The last section of data presentation used solely for displaying health information values entered by the user is statistics. This option allows users to view the average value of each information category entered for a specific week, month, or half a year. The user chooses the timeframe of theaverage calculation via a button selection and is then able to view averages for all healthinformation types.

d) Logs

The logs option of HealthTracker can be considered an additional functionality. This iswhere users may enter data that doesn't directly measure a certain aspect of their health, such as food intake, physical activity, medications, orother miscellaneous items. The system still records everything entered in this section of the application. A user is able to make a data entry for date of their choosing. From the main screen of the logs section, a user is presented with a calendar view of all their data entries of log-typedata. They may edit this data or set up a reminderthat forces the system to display a notification at a certain time of a certain day, containing the information specified by the user. e) Risks

Finally, the risks option from the main interface of HealthTracker is how a user calculates diseaserisk percentages. After choosing this selection, the user then decides which disease they prefer tocalculate for, as well as the method of calculationto be used. The type of disease calculated, purpose, background, and restrictions for each calculation method are described after its selection by the user. If the user's healthinformation currently stored in the system doesn'tmeet the requirements for a specific calculation method, and the user attempts to calculate with this method, the system halts and displays a message describing why it will not allow for a calculation. Some calculation methods may prompt the user with questions during thecalculation process. The results of successful calculations are presented to the user in conjunction with a description of what the resultsmean, or the importance of the findings. Themethods of calculating each of these assessmentswithin HealthTracker is based upon each study'sofficial calculations or point systems. The risk assessments included in HealthTracker at thispoint in time are the following: Framingham 10- year Hard Coronary Heart Disease, Framingham10-year Cardiovascular Disease, Framingham 8- year Type 2 Diabetes, Framingham 6-year Fatty Liver Disease, Framingham 4-year Hypertension,American College of Cardiology Foundation 1- year Arteriosclerotic Cardiovascular Disease, QRISK 10-year Heart Attack or Stroke,FINDRISC 10year Type 2 Diabetes, Qdiabetes 10-year Type 2 Diabetes, and the ARIC 9-year Type 2 Diabetes risk assessment. Each of these risk assessments has been independently validated, and many are widely used in professional medical practices across the world.

\subsection{Healthvault cloud storage}

When a user opens HealthTracker for thefirst time on their mobile device, they will be asked to log in using their Microsoft account. If a user logs in with Microsoft, all following health information that is entered into HealthTracker will also be uploaded to Microsoft HealthVault. This is done automatically, whenever a user makes a health information data entry, and is notsomething that has to be executed by the user. This function increases the reliability of our system. In the case of a system crash or data corruption related specifically to the operating system of the smartphone being used to run HealthTracker, users will not lose any of their health information as long as they have previously synced their Microsoft account with HealthTracker. Because Microsoft HealthVault provides accessibility to user health information,this also means that users are able to view their health data on any device that is capable of running HealthVault, not just smartphones, increasing the scope of our system functionality.

\subsection{Risk assessment}

Our goal in designing the risk assessment portionof HealthTracker was to ensure the user wasaware of what was happening at each step of the process. The system calculates, using the most recent health information entries in the system, auser's percent risk of developing a chronic disease within a certain amount of years. The usermust first decide what disease to calculate their risk for and, like mentioned before, what methodthe system should utilize in calculating their risk.All calculation methods were extracted from research institutions that developed formulas for risk calculations based on scientifically backed data. A description of each method and what it calculates for is provided to the user before they confirm their selection. They are then shown what health information the system will pull fromtheir health data records and given a description of how that information will be used. The user must accept a confirmation message that states the system's calculations can't be treated as official medical records and may then view their calculated percent risk of developing the selected disease for the specific timeframe. This process isseparated into tabbed menu system, consisting offour different tabs, each containing their own step of the process. The first tab provides thebackground information of the calculation method. The second shows users the utilized health 
information and asks the user to fill out anyadditional questions that are necessary for calculations. The third tab gets the user's confirmation, while the fourth displays the resultsof the system calculations.

\section{Implementation}

HealthTracker acts as a mobile health datarepository and analysis tool, with auxiliaryfunction as an event diary. Through this, the implementation has been divided into foursections - each part with different activities andpurposes. The application executed on an Android smart device has no central server, theonly internet requirement being supplementarycloud storage using Microsoft HealthVault.HealthTracker was developed as well as designedin Java using Android Studio. The user-designercommunications gap was mostly avoided duringimplementation due to in-depth research and userstory creation; the end user was kept in mind.

\subsection{Main interface}

1) Home screen

As one enters the application, after already having created a system user and linking it with their Microsoft HealthVault account, they are greeted with a twelve-button home screen. The buttons have been split into three sections, the first being a dropdown menu for multi-user support; it is previewed by displaying the currentuser's name. The second section displays data entry choices for weight, glucose, blood pressure,cholesterol, and thyroid measurements. The nextsection, located on the bottom of the screen in a row, contains buttons relating to diary entries, data analysis, and settings. Each menu choice is represented using a button-specific title and greyscale icon. Figure 1 shows the main menu ona smart phone.

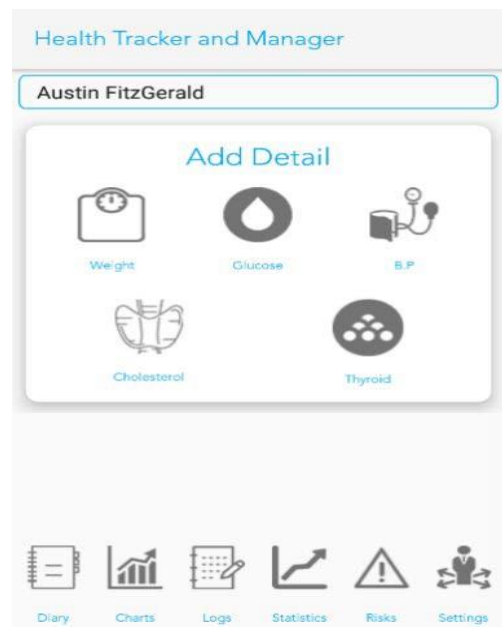

Fig. 1: Home Screen.

2) Settings

We found it imperative to keep accessibility in mind for all users, for that reason the applicationsupports multiple users and all measurable units can be changed. These choices allow comfortableusage in HealthTracker for people of different areas or professions. The settings activity also allows a user to set a reminder to record their readings at a specified time. The settings activityis shown in Figure 2 .

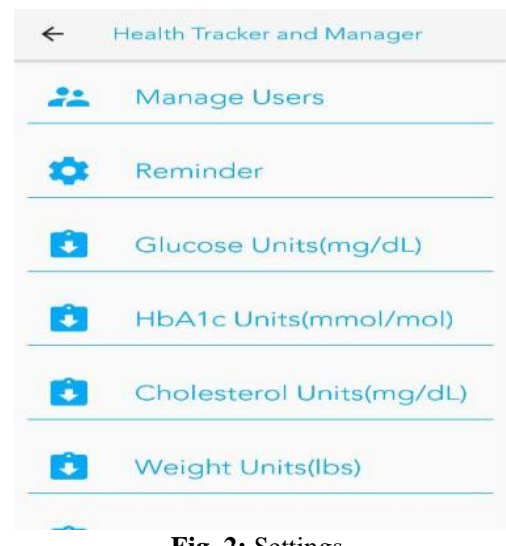

Fig. 2: Settings.

\subsection{Health data}

1) Detail Entry

A user can record five different data types: weight, glucose, blood pressure, cholesterol, andthyroid. A new weight entry contains five optional data fields: weight, fat percentage, abdomen circumference, waist circumference, and hip circumference. A glucose entry contains two optional data fields: glucose level and HbA1clevel; it also contains a scheduling dropdown forwhen the glucose level was taken, as well as the blood component (plasma or whole blood). Bloodpressure entries contain option data fields forsystolic and diastolic blood pressure along with aheart rate field. Each cholesterol entry contains five optional data fields: HDL, LDL, total cholesterol, and triglycerides. A thyroid entrycontains only one data field for a TSH level. Additionally, every data type entry has an optional note entry 
field and buttons to enter a custom date and time of the record; the default isthe current date and time. A detail entry for weight is shown in Figure 3.

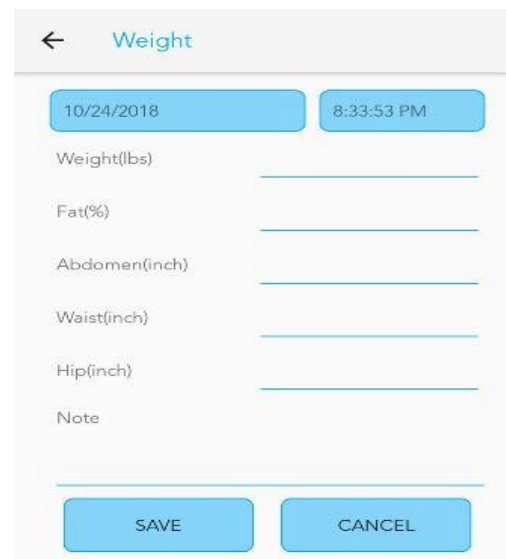

Fig. 3: Weight Detail Entry

2) Logs

The logs activity is implemented to display previously entered health data in a chronologicalsection-based format. It contains five tabs that coincide with all available data types: weight, glucose, blood pressure, cholesterol, and thyroid.Each tab lists the specified data type's entries in cards, where each card contains specific entry information. All applicable information for each data entry is displayed quantitively, with empty data fields shown as such. Units are shown as a user has chosen them in the settings, and numbersare converted if needed. Each data entry card includes a grayscale icon representing the chosendata type in order to provide extra accessibility. A data entry card can be clicked, which displays a dialog which focuses on the chosen entry and its specific data. In this, a user has the option to edit or delete the entry if they choose to do so.

3) Charts

Analyzing health trends can be difficult for layman users, and research has shown that graphsare advantageous in this regard. Once the charts option is chosen on the home screen, the user is brought to an activity containing more buttons, represented by titles and icons, for different charttypes. These include the main five data types as well as charts for two data types in one chart: weight and blood pressure, weight and glucose, weight and cholesterol, as well as weight and thyroid. Once the type is chosen, the system builds a chart with corresponding data. The data measurements are displayed on the y-axis, with the record date on the $\mathrm{x}$-axis. Each chart has a legend with the data type and complementary color. In the case of a multi-datatype chart, chartlines are presented with different colors.

4) Statistics

Along with monitoring health trends via charts, a user can also view quantitative statisticsfor each data type and its sub-sections. This activity is laid out in cards for each data type, each card contains corresponding averages for itsdata fields. A user can choose the time period in which to average data from using the following presets: week, month, and 6-month. If they wish to enter a custom time range, they can do so by entering their chosen number of preceding days. Once the calculate button is clicked, all data fieldsare populated with the average data for the giventime period. If no data has been entered for the specific data field during the given time period, such is shown. All results are shown with the settings-defined units and data is converted ifneed be. The statistics menu can be seen in Figure4.

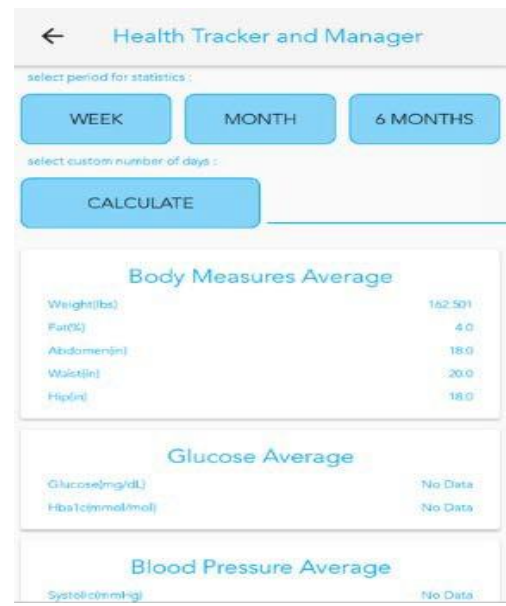

Fig. 4: Statistics.

\subsection{Diary}

1) Entry

The diary entry activity is also tab-based.Available diary entries include medications, food, exercise, and miscellaneous. Themedication tab includes a scheduling option, which opens a dropdown list for an expression ofwhen the medication was taken, e.g. "After Dinner". One then can choose the specific medication they wish to add through a dialog of saved medications. If a user wishes to add a new medication and the number of units taken, they can do so; each medication has a name and unit (mg, pills, puffs, or suppositories). If a user wishes to add a food entry, they can do so in a very similar way to adding a medication. The food entry tab contains a scheduling option as well as a saved food and its number of units, userscan add a new food with a specified name and unit type (grams or cups). The activity 
tab once again contains a scheduling option and a way to choose a saved activity or add a new one, the unitsfor this are minutes, miles, kilometers, or meters.Each diary entry type contains an optional note entry field, which is the only field for a miscellaneous entry, and buttons to enter a custom date and time; the default is the current date and time. A diary entry for medication is shown in Figure 5.

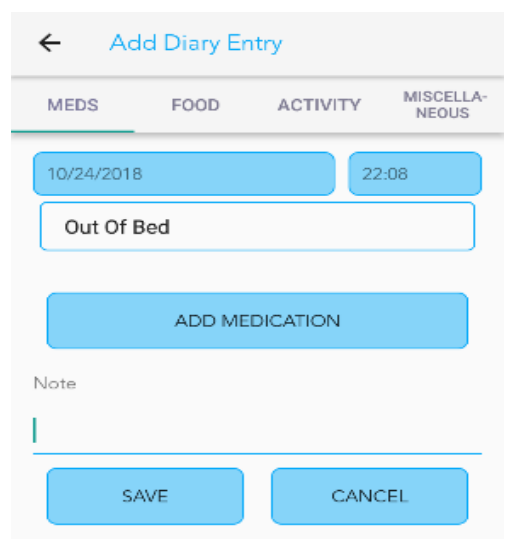

Fig. 5: Medication Diary Entry.

2) Viewing

Diary entries can be displayed in two ways, list orcalendar view, and are chosen by their complementary buttons. In list view, the usermust choose the type of diary entries to display, which are specified in a checkbox-listed dialog. They also must select a time period to display diary entries for, these are also specified in a dialog where the options are: all-time, 7-day, 14-day, 30-day, and 90-day. Once these constraints are met, diary entries are displayed inchronological order. Each diary entry is displayedin a card which has a color-coded stripe depending on the entry type. Each card is populated with the entry information: medication/food/exercise type, its quantity and units, the scheduling option, as well as the date and time of the record. Miscellaneous entries onlycontain the note entered upon creation as well asthe date and time. A user can click on a diary entry card to edit it or delete it. If the user wishes,they can also display their diary entries via a calendar. When the monthly view button is clicked a calendar is displayed, the current date ishighlighted but a user may choose any date that they wish. Upon clicking a date, the application displays a dialog containing diary entry cards forthe specified date; these cards are displayed in thesame way and have the same functionality as thecards in list view. If a date has no diary entries, none are displayed.

\subsection{Risk assessment}

Upon entering the risk assessments activity, the application displays a scrollable list of buttons which correspond to different chronic illness risk factor assessments. The user may choose any of these assessments by clicking its button. Each risk assessment's implementation is sectioned into four pages. The first pages consistof a disclaimer (Figure 6) which explains which chronic illness it is assessing for, the time periodin which the risk assessment is valid for, and anygeneral constraints such as an age limitation. Along with the disclaimer, any sources for the risk assessment are listed. There is an "Okay" button which a user may click when they are ready to continue if one does not meet the generalrequirements listed in the disclaimer they will notbe allowed to continue to the next page and will be notified as such. The next page can be reachedby clicking the forward arrow icon, which changes tints when it is allowed to be clicked. A user can always go back to the previous page, unless already on the first page. The second page(Figure 7) contains a scrollable list of questions required for the specific risk assessment that are not found in the user's previously entered health data. These questions also contain requirements for the assessment such as previous diagnoses ofdiseases. At the bottom of the page is a "Done" button, and if all requirements are met the forward arrow icon darkens and the next page is unlocked. The third page (Figure 8) is specific toquantitative health data that a user has previouslyentered, the specific data types displayed are different for each assessment. Each data type is shown via a title and has a drop-down menucontaining all valid data entries for that type. Each data entry contains the date of the record aswell as the measure and units which correspond with the chosen unit option in the settings activity. At the top of the page is a disclaimer warning the user to choose data within twenty- four hours of each other for best results. Once the"Done" button is clicked for this page, the forward arrow icon is automatically darkened, and the next page is unlocked; there are no more requirements to be met. The fourth and final page(Figure 9) contains a statement reaffirming the risk assessment description and time period it is valid for. The user can then press the "Calculate"button, which populates text with their percentage risk of the chosen chronic disease. If there were any issues with the user's data this textwill not be populated, the user is notified as such.Also, some risk assessments require additional disclaimers to be given upon the display of their results, these are displayed in a dialog after the "Calculate" button is clicked. 


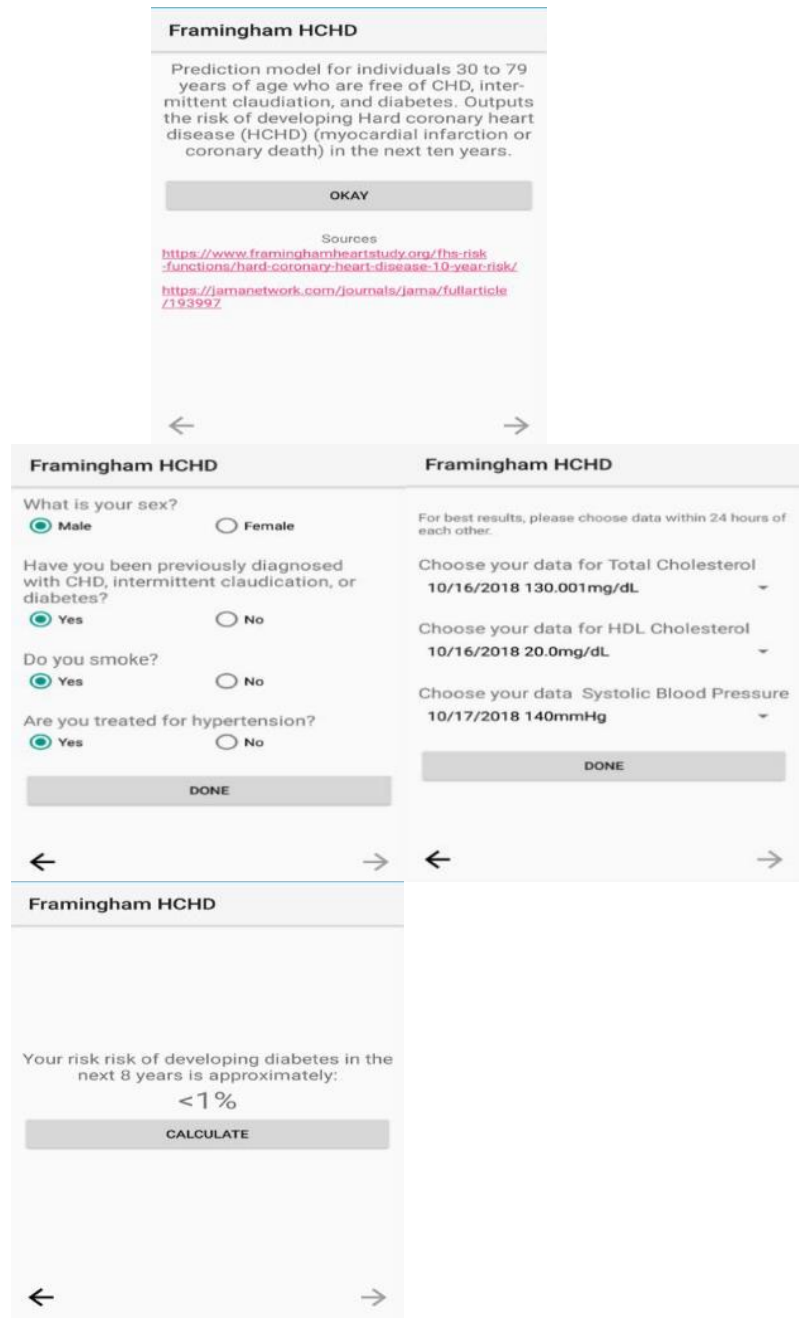

Fig. 6: Information Questions and Data Selection.

\section{Future work}

We believe HealthTracker can further developinto a system that benefits an even larger amount of people. As we uncover more algorithms for calculating a person's disease risk, we plan to addthese methods as more functionalities of risk assessment. Additional health data types may be necessary in order to calculate for a wider varietyof chronic diseases. This is a small matter to dealwith, seeing that we already have a foundation fordata acquisition as well as risk assessment.

Currently, it is clear that HealthTracker is effective in storing health information manually entered by a user. In the future, we envision the system interacting with sensor hardware, worn bya user, that retrieves health informationautomatically at set intervals of time. Whenever new health information is stored a user or caregiver could remotely monitor health statuseswithout having to repeatedly gather and enter thatperson's health information. A patient connectedto a wireless sensor system that is linked with HealthTracker could be periodically checked on by their caregiver in order to ensure the status of their health without being in direct contact with the individual without being in physical contact with the individual 1. Since HealthTracker is run on mobile devices, the caregiver may accomplishthis task from a distance as they pursue other daily objectives, only intervening with andassisting the patient if issues are detected byHealthTracker.

\section{Contributions and conclusion}

Instead of developing another application to add to the plethora of mobile operations that perform one specific task, we devised a system to assistand guide people with several aspects relatedto their overall physical health. Inspired by the work of previous software development teams and medical professionals, we imagined a multi- functional health monitoring system called HealthTracker. It would be capable of recording, storing, and analyzing user health information to assist those that live with uncertainty in relationto their health status and desire a way to discover more about what is happening within their body.

\section{References}

[1] Utilization Patterns Among Individuals in an Incentivized Program for Healthy Behaviors. Journal of Medical Internet Research,18(11). https://doi.org/10.2196/jmir.6371.

[2] Kim, J. Y., Wineinger, N. E., Taitel, M.,Radin, J. M., Akinbosoye, O., Jiang, J., Steinhubl, S. (2016). Self-Monitoring

[3] Lane, ND. Mohammod, M. Lin, M. et al.BeWell: A Smartphone Application to Monitor, Model and Promote Wellbeing. Computer Science Department Dartmouth College. 2011. https://doi.org/10.4108/icst.pervasivehealth.2011.246161.

[4] Ly, Z. Xia, F. Wu, G. Yao, L. Chen, Z. iCare: A Mobile Health Monitoring Systemfor the Elderly. Dalian University of Technology. 18 Dec, 2010.

[5] "How Does Health Spending in the U.S.Compare to Other Countries?'Peterson-Kaiser Health System Tracker. 
[6] Singh, C. (2018, October 12). Most Popular Android Versions 2018 (Always Updated List). Retrieved from https://fossbytes.com/most-popularandroid-versions-always-updated/

[7] Urzi, D., Stapleton, P., \& Chatwin, H.(2016) The use of a daily diary system to promote self-monitoring and improve health-related identity and selfefficacy. Graduate Journal of Sport and Exercise \&Physical Education Research, 4: 14-28.

[8] Alkhushayni, Suboh, and Susan McRoy."mHealth technology: towards a new mobileapplication for caregivers of the elderly living with multiple chronic conditions (ELMCC)." Proceedings of the 6th International Conference on Digital Health Conference. 2016. https://doi.org/10.1145/2896338.2896350.

[9] Alkhushayni, Suboh M. "mHealth technology: Towards a new persuasive mobile application for caregivers that addresses motivation and usability." (2016). https://doi.org/10.1145/2896338.2896350.

[10] Alkhushyni, Suboh M., M. Alzaleq Du'a,and Nadine L. Gadjou Kengne. "Blockchain Technology applied to ElectronicHealth Records." Proceedings of 32nd International Conference on. Vol. 63. 2019.

[11] Kruse, Ryan, and Suboh Alkhushayni. "Identifying regional COVID-19 presence earlywith time series analysis." IOP SciNotes 1.2 (2020): 024003. https://doi.org/10.1088/2633-1357/aba739.

[12] Alkhushayni, Suboh M., Daniel C. Zellmer, and Ryan J. DeBusk. "Text emotion mining on Twitter." IOP SciNotes 1.3 (2020): 035001. https://doi.org/10.1088/2633-1357/abc01e.

[13] Alkhushyni, Suboh M., M. Alzaleq Du'a, and Nadine L. Gadjou Kengne. "Blockchain Technology applied to Electronic Health Records." Proceedings of 32nd International Conference on. Vol. 63. 2019

[14] Al-zaleq, Du. "Optical Fiber Communication with Vortex Modes." (2019).

[15] Suboh Alkhushayni, Taeyoung Choi, Du'aAlzaleq, "Data analysis using representation theory and clustering algorithms", InternationalJournal of Engineering and Technology, 2020. https://doi.org/10.14419/ijet.v9i4.31234. 